\title{
Extrusion in Hydroenvironment in Laboratory Conditions
}

\author{
Ján Moravec ${ }^{1, *}$ \\ ${ }^{1}$ University of Žilina, Department of Technological Engineering, Univerzitná 8215/1, 01026 Žilina, \\ Slovakia
}

\begin{abstract}
The application of liquid environment in forward extrusion is described in this paper. Theoretical part states findings from fluidic mechanics applied in the experimental part. Design solution of the tool is also presented. The experiments were done in laboratory conditions. The findings are stated with their limitations.
\end{abstract}

\section{Theoretical findings}

According to Figure 1 points $N$ and $N^{\prime}$ define the axis of vertical cylinder. Vertical height difference between points $N, N^{\prime}$ is $h$. All pressures perpendicular to the surface, here vertical, form a neutral resultant. The weight $\rho d S h$ contained in the cylinder operates in vertical direction, then pressures $p_{N} d S$ and $p_{N} d S$. First two forces operate down, the third operates up $[1,2,3]$.

Liquid in the cylinder is in balance according to: $\rho d S h+p_{N^{\prime}} d S-p_{N} d S=0$. When divided by surface element $d S$, we get: $p_{N}=p_{N^{\prime}}+\rho h$. (1)

The difference of the specific pressures of two different points inside the liquid equals to the weight of vertical column of liquid, which has a unit of surface as a base and a difference of height contour of both points. The basic law of hydrostatics applies in any values of pressure $p_{N^{\prime}}$ a $p_{N}$ in equation (1). If pressure $p_{N}$ rises by value $\Delta p, p_{N^{\prime}}$ rises also by $\Delta p$. The increment of pressure is spreading evenly in all directions, because both considered points do not have to be on a common perpendicular $[4,5]$.

\footnotetext{
* Corresponding author: Jan.Moravec@ffstroj.uniza.sk
} 


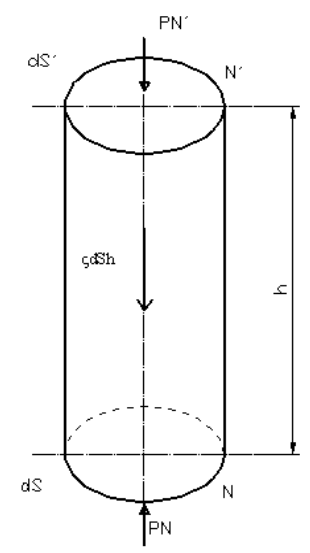

Fig. 1. Proportions in the vertical cylinder.

Let $M$ be a point of surface of solid planar wall, which the fluid is touching in balance according to Figure 2. We set out an area element $d S$ around point $M$. The liquid effects the wall with pressure force $d P$, solid wall reacts to liquid with force, which is equal, but opposite direction. Next we consider elementary cylinder with base $d S, d S^{\prime}=d S$ with surface perpendicular to the wall. In $\mathrm{M}^{\prime}$ point as a centre of surface $d S^{\prime}$ and infinitely close point $M$ let $\mathrm{p}$ be the pressure of liquid. If the outer forces affecting the liquid contained in the elementary cylinder project themselves into the axis of the cylinder and neglecting volume forces, which are infinitely small, third order against the surface forces, we get: $p d S-d P=0$, $d P=p d S$. (2).

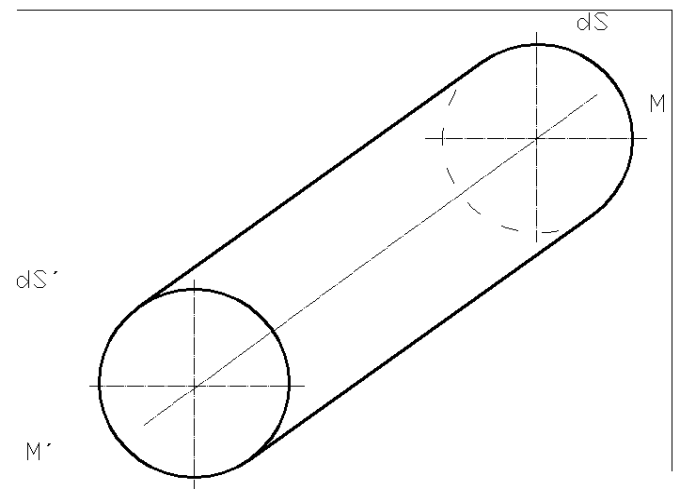

Fig. 2. Contact of the elementary cylinder with the planar wall.

Compressibility of the liquid is in general considered null. Although the molecules of the liquid move easily, liquids are noticeably compressible only by high pressures and we can say, that liquids are almost inelastic. The change of volume relative to original volume is proportional to change of pressure: $\Delta V / V \sim \Delta p_{p}$. If: $p$ - change of pressure affecting the liquid, $\beta$ - compressibility of liquid, $\Delta V$ - change of volume with rising pressure, $V$ - volume of the liquid, then applies: $\Delta V=\beta \Delta p V(3)$.

Using appropriate experimental methods, we can specify tensile-deformation condition in any place (point) of the deformation zone. Integral outputs generate energo-force parameters, 
which are used in dimensioning the tool and determining size of the forming machine. The degree of plastic deformation (reduction, ductile ratio, removal of material, etc.), can be defined as a sum of small intensities of deformation, by which the considered element of formed material goes through in its flow - movement through focal spot of deformation. It can be expressed with equation: $\lambda=E d t$, (4), where: $\lambda$ - degree of shear deformation in time frame of $0-t, E-$ intensity of shear deformation velocity.

$$
E=\sqrt{ } J_{2}\left(D_{\xi}\right)=\sqrt{ } 2 / 3 \sqrt{ }\left(\xi_{x}-\xi_{y}\right)^{2}+\left(\xi_{y}-\xi_{z}\right)^{2}+\left(\xi_{z}-\xi_{z}\right)^{2}+3 / 2\left(\tau_{x y}^{2}+\tau_{y z}^{2}+\tau_{z x}^{2}\right)
$$

In equation (5): $\xi_{x}, \xi_{y}, \xi_{z}$ - elements of velocities into linear deformations,

$\tau_{x y}, \tau_{y z}, \tau_{z x}$ - elements of shear deformations velocities.

For the flow line - axial, internal, surface it is necessary to count in the size of $\lambda$ and $E$ in between $t=0$, to $t=t_{K}$, therefore from the entry of the line into the focal point of deformation. We know the tensile condition of formed object when it is possible to determine elements of tensions $\sigma_{x}, \sigma_{y}, \sigma_{z}, \tau_{x y}, \tau_{y z}, \tau_{z x}$, or the main tensions $\sigma_{1,} \sigma_{2}, \sigma_{3},\left(\sigma_{1} \geq \sigma_{2} \geq \sigma_{3}\right)$ in the considered element.. Any expression containing these elements of tension is called tensile status indicator. Nádai - Lode coefficient of tension is preferred. It was found by verification, that this coefficient does not affect the limit plasticity $\lambda_{p}$ very much. A more appropriate dependence from shape indicator $k_{t}=\sigma_{o k t} / \tau_{\text {okt }}$ was determined experimentally. In equation: $\sigma_{o k t}$ is octaedric perpendicular tension, $\sigma_{\text {okt }}=1 / 3\left(\sigma_{1}+\sigma_{2}+\sigma_{3}\right) . \tau_{\text {okt }}$ is octaedric shear tension $\tau_{\text {okt }}=1 / 3 \sqrt{ }\left(\sigma_{1}-\sigma_{2}\right)^{2}+\left(\sigma_{2}-\sigma_{3}\right)^{2}+\left(\sigma_{3}-\sigma_{1}\right)^{2}$.

Generally, every process of plastic deformation can be defined as a constant process of formation, spreading and closing (for example by welding) of submicro and micro defect. If $\varphi$ is function of plasticity reserve, which has a starting status of material $=0$, according to Kolmogorov [2]:

$$
\varphi=\int_{0}^{t} B(t) \frac{E(t)}{\lambda_{D}\left[k_{z}(t)\right]} d t \quad\left(\text { a) } \quad \varphi=\int_{0}^{t} B(t) \frac{E(t)}{\lambda_{D}\left[k_{z}(t)\right]} d t<1\right.
$$

In relations $B(t)$ the quantity is dependent on the monotony of forming process. In monotonous processes, respectively almost monotonous, $B(t)=1$. In strongly monotonous processes, $B(t)>1$. $E(t)$ is function of time. In case of $(3 \mathrm{~b})$, there will be no material breach, when shearing $\varphi=1$ is desirable $[6,7,8,9]$.

\section{Construction of tool}

Using findings in the previous paragraph, a forming tool for forward extrusion was constructed - pic. 3. Basically it is a full metal tool, therefore by well-established criteria it should present a conventional forming technology. When replacing the extruder by liquid body, it is no longer a full metal tool. We can consider this „modified“ tool as an unconventional extruding construction. Active part (extruder) function was taken over by liquid body, and the extruder became a piston. Although the liquid is incompressible (as is generally assumed), it is still a hundred times more compressible than steel. Compressibility of liquid can be described by a volume compression coefficient $\beta_{V}$ or by elasticity modulus $k=1 / \beta_{V}$.

The volume conversation act applies in comparison of compressibility of water and steel at $t=20^{\circ} \mathrm{C}$. 
Water:

$$
\Delta V=-V \frac{\Delta p}{K}=-V \frac{\Delta p}{\frac{1}{100} E}=-100 V \frac{\Delta p}{E}=100 \Delta V
$$

Steel: $\Delta V=-V \Delta p / E$, where $\mathrm{E}=2.15 .10 \mathrm{~Pa} . k=1 / 100 E$.

Active tool parts are made of class 12 steel without heat treatment. The tool was used in laboratory conditions, so following experimental verification respects this fact fully.

\subsection{Strain on cavity and extrusion die body}

We can consider the extrusion die as a thick walled vessel - Figure 3. Radial and peripheral strain is present.

$$
\begin{aligned}
& G_{r}=\frac{p a^{2}-p_{o} b^{2}}{b^{2}-a^{2}}-\frac{\left(p-p_{o}\right) a^{2} b^{2}}{\left(b^{2}-a^{2}\right) r^{2}} \\
& G_{t}=\frac{p a^{2}-p_{o} b^{2}}{b^{2}-a^{2}}+\frac{\left(p-p_{o}\right) a^{2} b^{2}}{\left(b^{2}-a^{2}\right) r^{2}}
\end{aligned}
$$

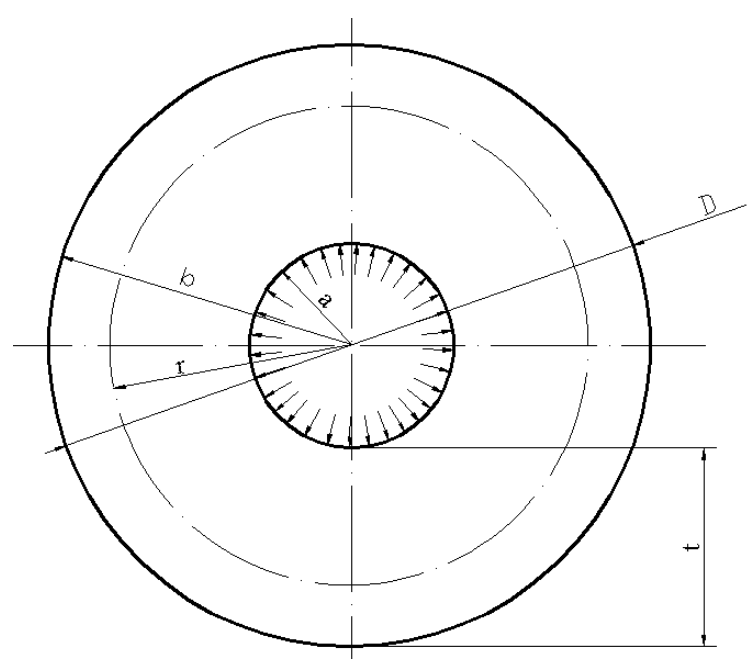

Fig. 3. Diagram for derivation of extrusion die strain.

The pressure in liquid column on the inside surface is: $p=\gamma . H$ ( $H$ - height of liquid column). Overpressure causes peripheral strain on the inner surface, for which the active parts needs to be dimensioned:

$$
\sigma_{t \max }=\frac{p\left(a^{2}+b^{2}\right)-2 p_{o} b^{2}}{b^{2}-a^{2}} \leq \sigma_{t d o v}
$$

if $p_{o}=0$, we can determine necessary outer radius of the part: $b=a \sqrt{\frac{\sigma_{d o v}+p}{\sigma_{d o v}-p}}$ 
These realities need to be considered with concrete constructional solution of the real extrusion tool for hydroforming.

\section{Experimental work}

The tool for forward extrusion was verified in laboratory conditions, as shown in Figure 4. In first verification, a model material was extruded using classic technology, without application of liquid for second experiment a liquid (oil) was poured between inserted intermediate material and a face of the extruder (piston) and using liquid body the model material was formed. $5+5$ specimens were made.

We can determine the forming pressure for forward extrusion according to [10]:

$p=k_{1}-\sqrt{ } k_{2}\left(k_{3}-q_{2}\right)\left(\mathrm{N} / \mathrm{mm}^{2}\right)$, where $k_{1}, k_{2}, k_{3}$ constants dependent on total carbon percentage in steel,

$q_{2}=d^{2}-D^{2} / d^{2} 100 \%$ (cross reduction of area), $d$ - beginning, $D-$ terminal diameter.

According to stated relation and considering real process with application of liquid, it is quite obvious that in this method a maximum quality seal is necessary. The technological process in the second method differs from the conventional one by filling the cavity with pressure liquid, thus inserting intermediate material into the cavity od extrusion die, filling the cavity with liquid and inserting the extruder, the main course of process and after its completion, pulling out the extruder and removing the liquid follows.

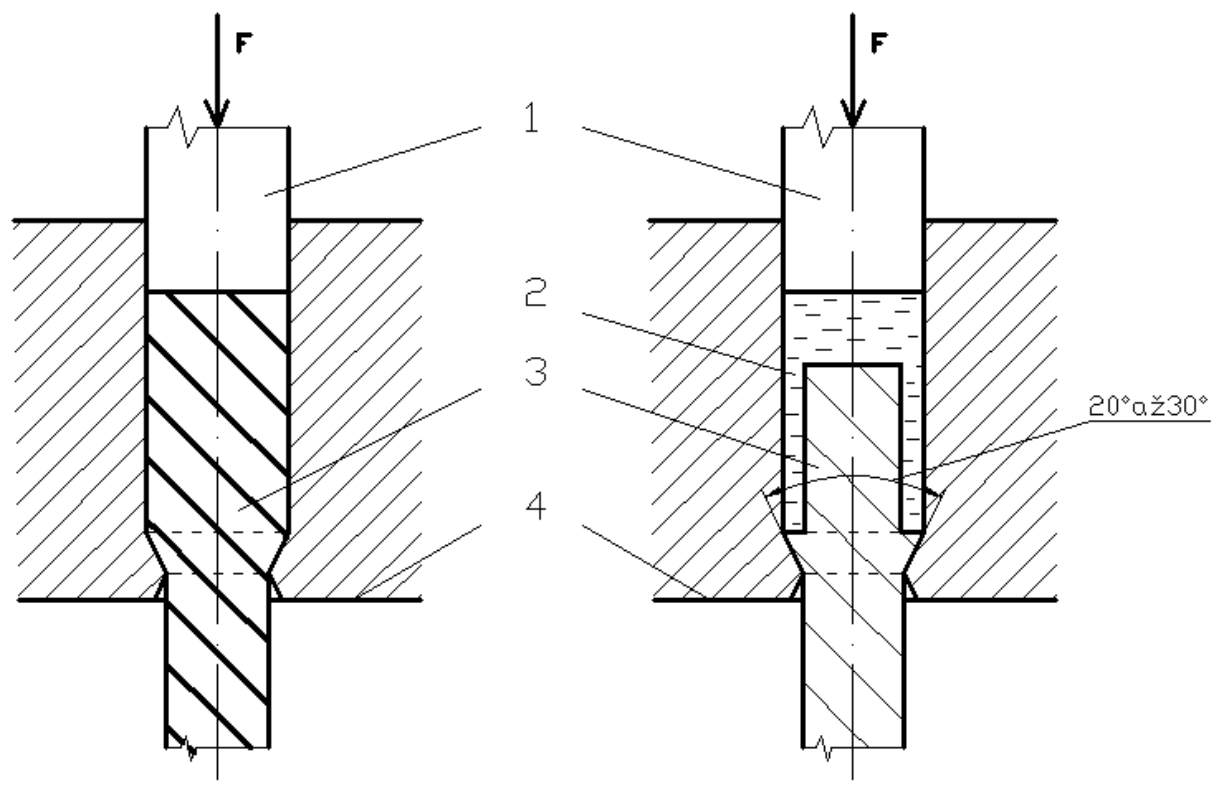

Fig. 4. Two conditions of the experiment: without and with the application of liquid. 1 - extruder (piston), 2 - liquid, 3 - formed material, 4 - extrusion die. 

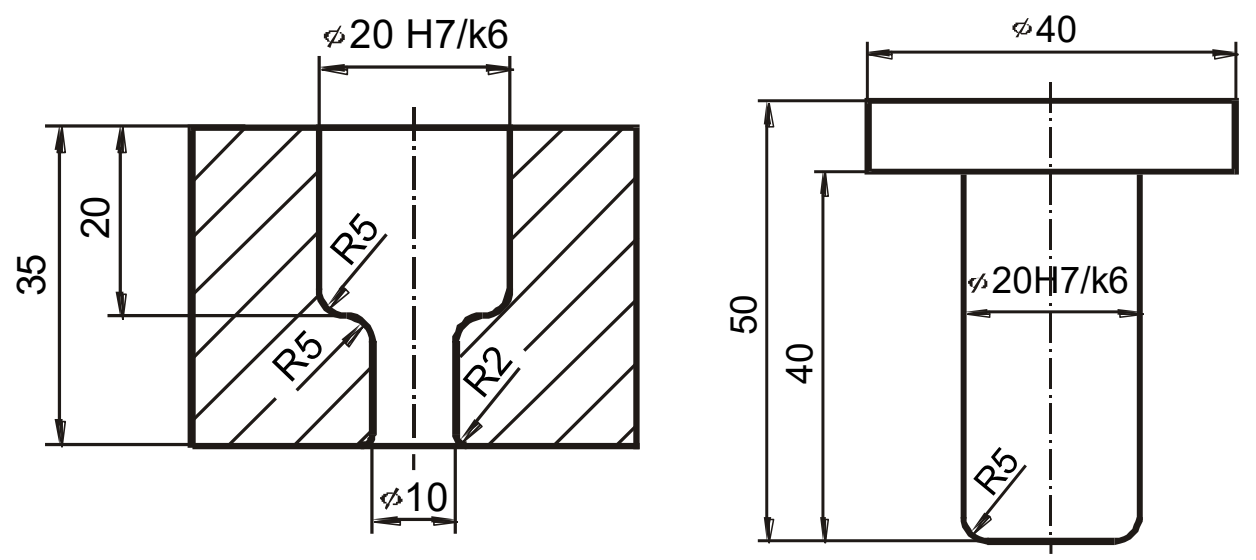

Fig. 5. Extrusion die (left) and extruder.

The photograph of the tool is in Figure 6.



Fig. 6. Experimental tool.

\section{Results and findings}

We can summarize them into these points: with pressing the extruder on the oil, process friction is reduced, angle of the extrusion die is lesser and quality of the product is far superior.

\section{Discussion}

Essential condition of optimal usage of formed material in the forming process is the forming tool, which should provide increased limit metal plasticity [11, 12]. It is about configuration of the forming process the way this condition is maximally satisfied. Limit plasticity of specified state and kind of material is not constant quantity, but is dependent on tension 
status, deformation velocity and temperature of the process. Forming processes can be deliberately influenced for:

- preventing state of limit plasticity in critical spots of deformation area, focus of deformation,

- achieving maximal usage of plasticity in forming process,

- designing forming process from the perspective of optimal plasticity usage of formed metal.

The diagram of hydrostatic extrusion through conical extrusion die is on pic. 7. In this method, the deformation focus is concentrated into the conical part of the extrusion die, where the variable factors are: extrusion ratio $-\kappa=D^{2} / d^{2}$, angle of the extrusion die $-2 \alpha$ and extrusion process velocity. If we consider the minimal extrusion pressure $p$ as an indicator, then $\partial \mathrm{p} / \partial \alpha=0$, it is possible to transfer the dependencies into charts $p$ - $\alpha$. With $p_{\min }$ and 2 $\alpha=45^{\circ}$, with increasing extrusion ratio was transferred to higher $\alpha\left(2 \alpha=70^{\circ}\right)$. When extruding materials with lower plasticity (dural, molybdaen, high speed steel), the optimal pressure angle of the extrusion die does not guarantee compact products (transversal or tree fractures going deep are visible on the surface). By decreasing the deformation degree and increasing $\alpha$ leads to formation of internal axial fractures. Courses of $\lambda$ and $k_{t}$ are important and crucial for assessing the level of tool construction from the perspective of product quality. The value of $\lambda$ from the entry to the deformation focus to its exit rises, this rising is almost linear for lower values of $\kappa$, especially with fibres around the wall of the extrusion die. With higher values of $\alpha$ and $\kappa>5, \lambda$ significantly rises in the centre of the deformation focus. Course of $k_{t}$ is similar, but at higher $\kappa$ a $\alpha$ we get to area of $k_{t}>0$, because in this condition $\lambda$ is higher. From equation (6) comes the probability of fracture formation closely under the surface.

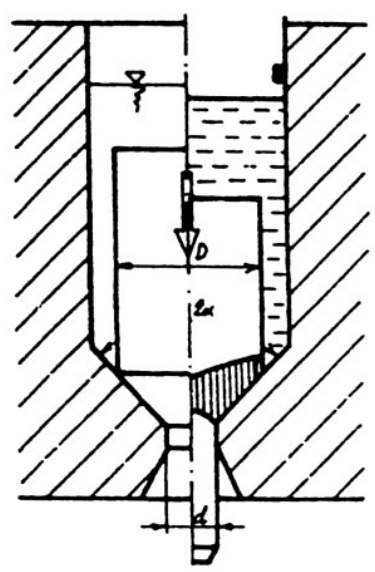

Fig. 7. Diagram of hydrostatic extrusion with focus of deformation shown.

\section{Conclusion}

Optimisation of forming processes based on their mathematization is the carrying science technologic trend of following research in forming. Modern, computerised forming files with optimized technology and integrated operation can be built on these principles. Analyses and their implementation into the wide spectrum of forming problematics is a necessary premise for successful research. Physics as a science field of activity is an inexhaustible source of information, from which we can draw almost without limits. 


\section{References}

1. W.F. Hosford, Metal Forming, Mechanics and Metallurgy, (2011)

2. V. Boljanovic, Sheet Metal Forming Processes and Die Design, (2004)

3. S. Kundar, Technology of Metal Forming Processe, (2008)

4. Z. Marciniak, Theory of sheet metal forming, (1964)

5. Z. Marciniak, et al. Mechanics of Sheet Metal Forming, (2005)

6. R. Pearce, Sheet Metal Forming. (1991)

7. Metal Forming Handbook, Schuler GmBH Göppingen, (2009)

8. J. Moravec, Unconventional metal forming technologies, (2011)

9. J. Moravec, $18^{\text {th }}$ Symp. on Anemo., (2003)

10. J. Moravec, Contribution to the problem of hydroforming, HaP, (2006)

11. J. Moravec, J. Bílik, (2017) Forming machines and tools, (2017)

12. J. Moravec, The construction of a hydrostatic molding tool, Technolog IV. (2012) 